\title{
Water immersion methods do not alter muscle damage and inflammation biomarkers after high-intensity sprinting and jumping exercise
}

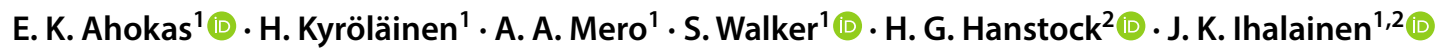

Received: 24 April 2020 / Accepted: 21 August 2020 / Published online: 2 September 2020

(c) The Author(s) 2020

\begin{abstract}
Purpose The aim of this study was to compare the efficacy of three water immersion interventions performed after active recovery compared to active recovery only on the resolution of inflammation and markers of muscle damage post-exercise. Methods Nine physically active men $(n=9$; age 20-35 years) performed an intensive loading protocol, including maximal jumps and sprinting on four occasions. After each trial, one of three recovery interventions (10 min duration) was used in a random order: cold-water immersion $\left(\mathrm{CWI}, 10{ }^{\circ} \mathrm{C}\right.$ ), thermoneutral water immersion (TWI, $24{ }^{\circ} \mathrm{C}$ ), contrast water therapy (CWT, alternately $10{ }^{\circ} \mathrm{C}$ and $38{ }^{\circ} \mathrm{C}$ ). All of these methods were performed after an active recovery (10 min bicycle ergometer), and were compared to active recovery only (ACT). $5 \mathrm{~min}, 1,24,48$, and $96 \mathrm{~h}$ after exercise bouts, immune response and recovery were assessed through leukocyte subsets, monocyte chemoattractant protein-1, myoglobin and high-sensitivity C-reactive protein concentrations.

Results Significant changes in all blood markers occurred at post-loading $(p<0.05)$, but there were no significant differences observed in the recovery between methods. However, retrospective analysis revealed significant trial-order effects for myoglobin and neutrophils $(p<0.01)$. Only lymphocytes displayed satisfactory reliability in the exercise response, with intraclass correlation coefficient $>0.5$.

Conclusions The recovery methods did not affect the resolution of inflammatory and immune responses after high-intensity sprinting and jumping exercise. It is notable that the biomarker responses were variable within individuals. Thus, the lack of differences between recovery methods may have been influenced by the reliability of exercise-induced biomarker responses.
\end{abstract}

Keywords Cold-water immersion · Thermoneutral water immersion · Contrast water therapy $\cdot$ Recovery $\cdot$ Inflammation

$\begin{array}{ll}\text { Abbreviations } \\ \text { ACT } & \text { Active recovery only } \\ \text { CK } & \text { Creatine kinase activity } \\ \text { COR } & \text { Serum cortisol }\end{array}$

Communicated by Michalis G Nikolaidis.

Electronic supplementary material The online version of this article (https://doi.org/10.1007/s00421-020-04481-8) contains supplementary material, which is available to authorized users.

J. K. Ihalainen

johanna.k.ihalainen@gmail.com

1 Biology of Physical Activity, NeuroMuscular Research Center, Faculty of Sport and Health Sciences, University of Jyväskylä, P.O Box 35, 40014 Jyväskylä, Finland

2 Swedish Winter Sports Research Centre, Department of Health Sciences, Mid Sweden University, Östersund, Sweden
CWT Contrast water therapy

CWI Cold-water immersion

hsCRP High-sensitivity C-reactive protein

MCP-1 Monocyte chemoattractant protein-1

TWI Thermoneutral water immersion

WBC Leukocytes

\section{Introduction}

High-intensity exercise results in numerous physiological perturbations and insufficient recovery from these perturbations might result in suboptimal performance and training quality during subsequent training sessions, while chronic imbalance between training stress and recovery might lead to suboptimal training adaptations (Bleakly \& Davison 2010). Water immersion methods are nowadays popular recovery methods among athletes as well as the general population. 
Immersion strategies can be divided into four major categories: cold-water immersion (CWI; $\leq 20^{\circ} \mathrm{C}$ ), hot water immersion (HWI; $\geq 36^{\circ} \mathrm{C}$ ), thermoneutral water immersion (TWI; $21-35^{\circ} \mathrm{C}$ ) and contrast water therapy (CWT; alternating CWI and HWI) (Versey et al. 2013). These recovery interventions may be used with the intention of attenuating delayed onset of muscle soreness and accelerating recovery from muscle-damaging exercise (Pournot et al. 2011; Versey et al. 2013; Dupuy et al. 2018).

Although the majority of peer-review papers have observed limited positive effects of immersion compared to control groups for recovery measures, there is substantial heterogeneity in loading protocols and recovery responses. To this end, meta-analytical data suggests potential small but positive effects of CWI (Bleakley et al. 2012; Higgins et al. 2017; Dupuy et al. 2018) and CWT (Higgins et al. 2017) on performance and/or perceptual recovery from high-intensity loading exercise. Furthermore, muscle soreness may also be attenuated by CWI (Bleakley et al. 2012; Dupuy et al. 2018) and CWT (Dupuy et al. 2018). A number of mechanisms have been suggested to account for the enhanced acute recovery associated with post-exercise water immersions, one being a blunted inflammation response (White et al. 2014).

On the other hand, post-exercise inflammation may play an important role in mediating exercise-induced adaptations (Brunelli and Rovere-Querini 2008; Chazaud et al. 2009); for example, macrophages stimulate myoblast proliferation and differentiation, and thus are involved in skeletal muscle regeneration and tissue repair (Chazaud et al. 2009). Consequently, it has been suggested that water immersion methods could attenuate potentially desirable inflammatory responses to muscle-damaging exercise (Eston and Peters 1999; Vaile et al. 2007; Stacey et al. 2010; Murray and Cardinale 2015). Chronic use of CWI or other water immersion methods has been suggested to ultimately attenuate training adaptations (Yamane et al. 2006, 2015; Roberts et al. 2014).

The majority of previous studies have reported that CWI (Pournot et al. 2011; de Andrade Bezerra et al. 2014) and other water immersion methods (Pournot et al. 2011) do not affect leukocyte, lymphocyte, neutrophil, monocyte and macrophage counts compared to passive or active recovery. Nevertheless, Stacey et al. (2010) found that, an hour after exercise, leukocyte and neutrophil counts were higher and lymphocyte counts were lower after CWI compared to active and passive recovery, which could be beneficial for shortterm recovery (Stacey et al. 2010). Furthermore, an animal study by Camargo et al. (2012) showed that the density of inflammatory cells (cell/100 $\left.\mu \mathrm{m}^{2}\right)$ in rats muscle tissue was lower after CWI than in passive recovery (Camargo et al. 2012).

There is a limited amount of research on the effects of water immersion methods on muscle damage and inflammation markers and conflicting results about the effect of the water immersion methods on leukocytes and leukocyte subsets. Therefore, the purpose of this study was to examine the effect of three different water immersion methods after active recovery and active recovery alone on leukocytes, leukocyte subsets, myoglobin, and monocyte chemoattractant protein- 1 concentration and recovery following an acute bout of high-intensity exercise. The hypothesis was that water immersion methods after an active recovery do not give any added benefits on the clearance of muscle damage and inflammation markers following active recovery.

\section{Methods}

\section{Participants}

Nine healthy, men (mean \pm SD: age $26 \pm 3.7$ y, body mass $78.6 \pm 11.6 \mathrm{~kg}$, height $1.81 \pm 0.09 \mathrm{~m}$, body fat: $15.8 \pm$ $4.0 \%$ ), undertaking regular physical activity, provided written, informed consent to take part in the study. Sample size was estimated based on previous studies (Stacey et al. 2010; Crystal et al. 2013; Broatch et al. 2014; Peake et al. 2017). Upon enrolment, participants completed a health questionnaire, as well as food and activity diaries that facilitated standardisation of nutritional intake and physical activity before, during, and after the trials until the last recovery measurements. Ethical approval for the study was obtained from the Ethical Committee of University of Jyväskylä, and the study was conducted in accordance with the Declaration of Helsinki.

\section{Study design and procedures}

The recovery protocol was designed to match typical recovery routines used by athletes and the purpose was to investigate the possibility of added benefit from water immersion methods compared to standard (active) recovery routines frequently used by athletes. A schematic representation of the protocol has been published elsewhere (Ahokas et al. 2019). Briefly, participants were randomly allocated to active recovery only (ACT), or active recovery followed by cold-water immersion (CWI), contrast water therapy (CWT) or thermoneutral water immersion (TWI) on each test day. All participants completed all four experimental conditions, separated by at least 2 weeks, each time with a different recovery method. Prior to the recovery intervention, participants performed several physical performance tests and an intensive loading protocol, which included jumping and sprinting. The recovery procedure included active recovery (10 min bicycle ergometer, Monark 839 E, Vansbro, Sweden; heart rate $120-140 \mathrm{bpm}$ ) accompanied by consumption of a recovery beverage (Gainomax, $40 \mathrm{~g}$ carbohydrates and 
$20 \mathrm{~g}$ protein) and followed the assigned recovery intervention. The fluid intake was controlled during the loading protocol and the first hour of recovery. White blood cells and differential cell counts, MCP-1 and biochemical markers of muscle damage were measured at baseline (PRE), and then at five timepoints following the loading protocol: $5 \mathrm{~min}$, $1 \mathrm{~h} 24 \mathrm{~h}, 48 \mathrm{~h}$, and $96 \mathrm{~h}$ post-loading (POST5, POST60, POST24, POST48 and POST96, respectively; Fig. 1).

\section{Loading protocol and performance tests}

On each of the four trials, participants performed an intensive loading exercise protocol (total duration $45 \mathrm{~min}$; Fig. 1), which included:

- $2 \times 5 \times 10$ unilateral long jumps (walking back between sets / 5 min rest between blocks)

- $2 \times 3 \times 60 \mathrm{~m}$ running:

- Set 1: the target speed was $95 \%$ of the maximum speed tested the same morning;

- Set 2: the target speed was $98 \%$ of the maximum speed;

- 2 min rest between repetitions / 5 min rest between sets.

- $2 \times 200 \mathrm{~m}$ run at maximum speed (5 min rest).

During exercise, heart rate was continuously recorded with a heart rate monitor (Polar V800, Kempele, Finland), and there were no differences between trials. The performance tests involved a single $30 \mathrm{~m}$ maximal sprint, maximum bilateral isometric leg press and countermovement jump. The performance test protocol has been reported elsewhere and there were no differences in performance test results following different immersions (Ahokas et al. 2019). Furthermore, creatine kinase (CK) and cortisol (COR) responses in relation to self-reported muscle soreness have been reported elsewhere (Ahokas et al. 2019), and are presented here in a posteriori reliability analyses.

\section{Water immersions}

Following active recovery and consumption of the recovery beverage, each participant was immersed underwater in a sitting position to the level of the xiphoid process for $10 \mathrm{~min}$ in a dedicated bath, while during the ACT condition the participants remained seated in an empty bath for $10 \mathrm{~min}$. CWI and TWI were continuously immersed in water temperatures of $10^{\circ}$ and $24{ }^{\circ} \mathrm{C}$, respectively. In CWT, the participants alternated immersion at $38{ }^{\circ} \mathrm{C}$ and $10{ }^{\circ} \mathrm{C}$ with five cycles of 1 min in each bath.

\section{Collection and analysis of blood samples}

To assess the immediate and prolonged immune responses to each loading protocol/recovery protocol, blood samples were drawn from an antecubital vein into EDTA tubes (Venosafe, Terumo, Belgium), and serum tubes (Vacuette, Greiner-Bio-One GmbH, Kremsmünster, Austria) at baseline and at $5 \mathrm{~min}, 60 \mathrm{~min}, 24 \mathrm{~h}, 48 \mathrm{~h}$ and $96 \mathrm{~h}$ after completing the loading protocol. Total white blood cell count and subgroups; neutrophils, lymphocytes and mixed cells (monocytes, eosinophils, basophils and immature precursor cells) were determined with an automated cell counter (Sysmex KX-21 N, TOA Medical Electronics Co., Ltd., Kobe, Japan). Serum samples were held for $30 \mathrm{~min}$ at room temperature before being centrifuged for $10 \mathrm{~min}$ at $2000 \times g$ at $4{ }^{\circ} \mathrm{C}$ (Megafuge 1.0 R, Heraeus, Germany). Serum was then stored at $-80{ }^{\circ} \mathrm{C}$ until analysis. Serum samples were analysed for high-sensitivity C-reactive protein (hs-CRP) using commercial chemiluminescence immunoassay techniques (Immulite $2000 \mathrm{XPi}$, Siemens Healthcare GmbH,

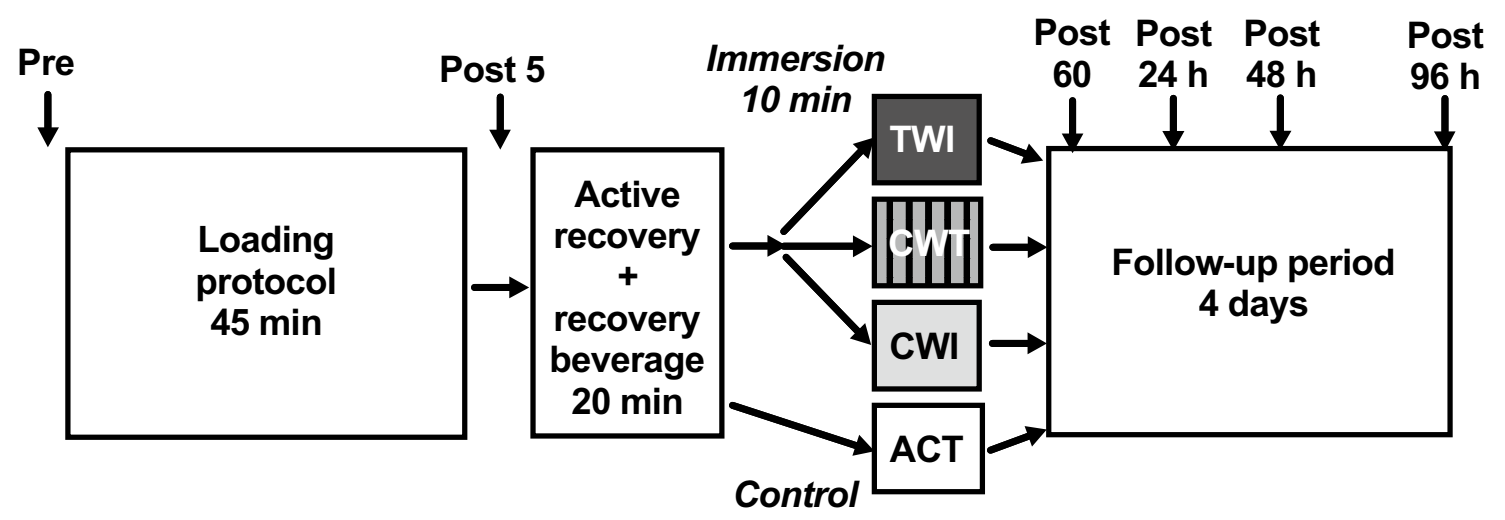

Fig. 1 Schematic of study design. Participants completed a standardised loading protocol followed by an initial recovery protocol (active recovery and recovery beverage). After the active recovery protocol participants completed one of three water immersion protocols (TWI temperate water immersion, $C W T$ contrast water therapy or $C W I$ cold-water immersion) or sat in an empty bath ( $A C T$ active recovery only, serving as control). Follow-up measurements were obtained at four additional timepoints after immersion (marked by arrows) 
Erlangen, Germany). Myoglobin and MCP-1 were analysed in serum samples in single using an enzyme-linked immunosorbent assay (ELISA) method with commercial reagents (R\&D Systems, Europe Ltd, Abingdon, UK) performed on an automated platform (Dynex DS2, Washington, USA). The detection limits and inter-assay coefficients of variation, respectively, were $0.1 \mathrm{mg} \mathrm{L}^{-1}$ and $10.1 \%$ for hs-CRP, $0.8 \mathrm{ng} \mathrm{mL}^{-1}$ and $10.2 \%$ for myoglobin and $3.9 \mathrm{pg} \mathrm{mL}^{-1} 6.2 \%$ for MCP-1.

\section{Statistical analyses}

Statistics were performed using commercial software packages (IBM SPSS version 24.0; SPSS Inc., Chicago, IL; Prism 8, GraphPad, La Jolla, CA). Data were checked for normality using Shapiro-Wilk tests; variables that did not satisfy the assumption of normality were log-transformed prior to analysis. Conventional statistical methods were used to obtain means, standard deviation (SD), 95\% confidence intervals (95\% CI), and Pearson's product moment correlation coefficients; for log-normally distributed variables, geometric mean and SD factors are reported. Two-way repeated-measures analysis of variance (ANOVA) was used to compare continuous variables between conditions across the pre- and post-loading and immersion timepoints. Due to two missing data points in the leukocyte analyses, a mixedeffects model using the maximum likelihood method was performed instead of a two-way repeated-measures ANOVA. Post-hoc tests were used to explore time effects compared to baseline within trials using Dunnet's correction for multiple comparisons. Data are reported and displayed as means and standard deviations (SD) for normally distributed data sets and geometric means and geometric SD for data sets that were log-transformed prior to analysis. Statistical significance was set at $p \leq 0.05$ but trends were explored with post-hoc tests when $p \leq 0.1$ for main effects.

\section{Results}

\section{White blood cell responses to recovery}

There were no differences in the magnitude of the exerciseinduced leukocyte response between trials. Furthermore, there were no differences in leukocytes or leukocyte subset concentrations during recovery between recovery interventions (Fig. 2). As expected, multiple significant time effects were observed between timepoints for all variables, illustrated in Fig. 2; for clarity, only differences from PRE for each trial are displayed. Leukocytes and all subsets increased in response to the loading protocol $(p<0.01$ for all variables, Fig. 2a). Leukocytes and mixed cells (Fig. 2c) generally returned to baseline around POST60, while lymphocytes
(Fig. 2b) increased at POST5 then decreased to below PRE values at POST60. Neutrophils generally remained elevated until POST60 (Fig. 2d). All leukocyte subsets had returned to baseline by POST24.

\section{Biomarker responses to recovery}

Two-way repeated-measures ANOVAs revealed no significant trial $\times$ time interactions in the blood biomarker responses (MCP-1, myoglobin, and hs-CRP) to the three water immersion methods compared to active recovery (Fig. 3). Trial $\times$ time interactions typically accounted for a very low percentage of total variance for all variables $(<3 \%)$. As expected, multiple time effects were observed between timepoints for each biomarker; for clarity, only differences from PRE are described. MCP-1 generally increased from PRE to POST5 and returned to baseline between POST60 and POST24 (Fig. 3a). hs-CRP generally increased between POST60 and POST24 and recovered to baseline between 48 and $96 \mathrm{~h}$ post-loading (Fig. 3b). Myoglobin increased from PRE to POST5 and continued to rise until POST60 (Fig. 3c). Myoglobin then decreased significantly from POST60 to POST24 and continued to recover until $48 \mathrm{~h}$ post-loading. No correlations were observed between biomarkers or biomarker responses.

\section{Exploratory descriptive analysis: variability of the immune response to a standardised loading protocol}

We noted that the variability of immune and inflammatory responses to the loading protocol was high within individuals, within the portion of the trial that was standardised on all visits. Therefore, we a posteriori investigated the variability of the exercise-induced muscle damage, inflammatory, and leukocyte responses by calculating the intraclass correlation coefficient (ICC) for biomarker concentrations and leukocyte counts at PRE, POST5 and the response magnitude between the two timepoints (fold change). Creatine kinase (CK) and cortisol (COR) responses to exercise, previously reported in Ahokas et al (2019), were also included in the a posteriori analysis. The ICC model selected was a two-way, mixed-effects model with absolute agreement (Koo and $\mathrm{Li}$ 2016). ICC values with $95 \%$ confidence intervals are displayed in Tables 1 and 2. Two-way repeated-measures ANOVAs were also used to explore trial-order effects across these two timepoints (Supplementary Figure S1 and Supplementary Figure S2). Trial-order effects were evident for COR (Trial 1 mean $>$ Trial 3 and 4, p < 0.01, Fig S1C), myoglobin (Trial 1 POST5 $>$ Trials $2-4, p<0.01$, Fig. S1F) and neutrophils (Trial 1 POST5 $>$ Trial 3 and 4, p < 0.01, Fig. S2). 

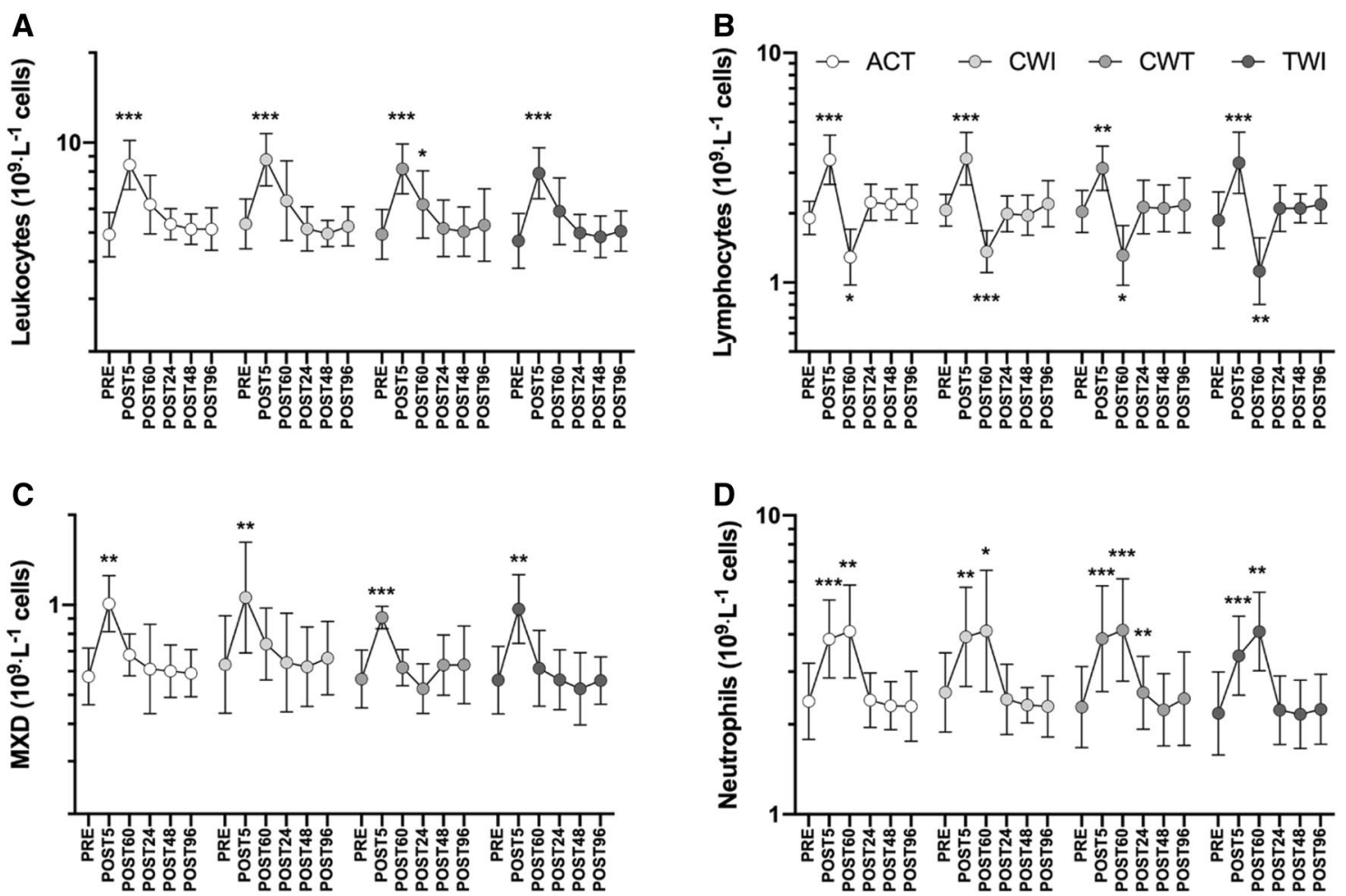

Fig. 2 Leukocyte (a), lymphocyte (b), mixed cell (c; MXD; monocytes, eosinophils, basophils and immature precursor cells) and neutrophil (d) responses to the loading protocol and recovery. Recovery interventions applied between POST5 and POST60; ACT active recovery only, $C W I$ cold-water immersion, $C W T$ contrast water

therapy and TWI thermoneutral water immersion. Data are geometric mean and SD factors displayed on a $\log _{10}$ scale. No differences in leukocyte or leukocyte subset responses were found between recovery interventions; however, effects of time are displayed for each subset as differences from PRE. $* p<0.05, * * p<0.01, * * * p<0.001$

\section{Discussion}

The main finding of this study was that water immersion methods did not alter circulating muscle damage and inflammation biomarkers after high-intensity sprinting and jumping exercise. Therefore, the water immersion methods used in the present study were not shown to give any added benefits on the clearance of inflammation, nor did they attenuate muscle damage and inflammation responses assessed with circulating biomarkers.

A bout of high-intensity loading triggers a transient inflammatory response comprising augmented white blood cell counts and stimulation of pro- and anti-inflammatory cytokine production (Freidenreich and Volek 2012). The physiological stress caused by exercise acts as a major stimulus for muscle fibre hypertrophy, and efficient repair of muscles requires a well-coordinated and controlled inflammatory response (Peake et al. 2010). C-reactive protein (CRP) is an acute phase reactant that can be used to monitor inflammation episodes (Brouwer and van Pelt 2015). In the present study there were no differences in hs-CRP between recovery interventions, although CRP responded to and recovered from loading in all groups. Previous studies on CRP have

reported either no difference (Ingram et al. 2009; Pointon et al. 2012) or decreased CRP levels after CWI compared to passive recovery (Brophy-Williams et al. 2011), and TWI (Ascensão et al. 2011). Physiological responses to water immersion methods have been suggested to be influenced by hydrostatic pressure and water temperature (Wilcock et al. 2006). The cold temperature of CWI is believed to enhance recovery by attenuating the inflammatory response to muscle-damaging exercise, as cold induces localised vasoconstriction (Mawhinney et al. 2017), which is suggested to reduce permeability of cellular, lymphatic, and capillary vessels, reducing fluid diffusion into the interstitial space (Eston and Peters 1999; Murray and Cardinale 2015). Furthermore, reduced fluid diffusion may assist in reducing acute inflammation and oedema (Cote et al. 1988; Yanagisawa et al. 2003; Stacey et al. 2010; Murray and Cardinale 2015). However, the results of previous studies (Pournot et al. 2011; Crystal et al. 2013; Broatch et al. 2014; de Andrade Bezerra et al. 2014) and the present study do not support these assumptions.

Exercise-induced muscle damage is triggered when individuals engage in new types of exercise, especially lengthening or eccentric contractions, which are conducted with a 

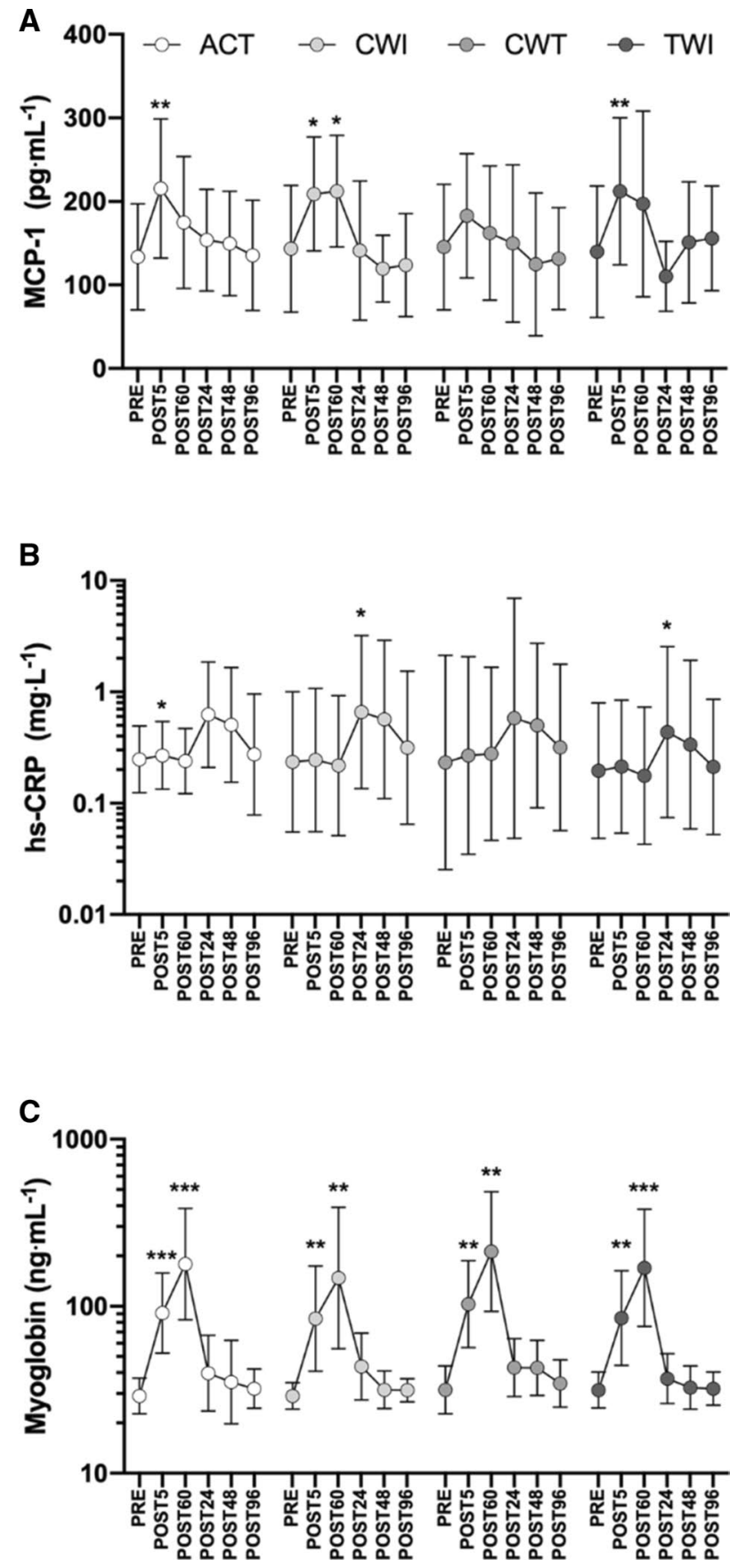

Fig. 3 MCP-1 (a), hs-CRP (b), and myoglobin (c) responses to loading and recovery. Recovery interventions applied between POST5 and POST60; ACT, active recovery only, CWI, cold-water immersion, CWT, contrast water therapy and TWI, thermoneutral water immersion. Data are geometric mean and SD factors displayed on a $\log _{10}$ scale. No significant differences in biomarker responses were found between recovery interventions; however, effects of time are displayed for each marker as differences from PRE. ${ }^{*} p<0.05$, $* * p<$ $0.01, * * * p<0.001$ large range of motion. The mechanisms implicated in exercise-induced muscle damage, inflammation, and hypertrophy have been comprehensively reviewed elsewhere (Wackerhage et al. 2019). Blood levels of myofibre proteins such as myoglobin and CK are often used to quantify exerciseinduced muscle cell injury (Warren et al. 1999). The present study did not observe any differences in myoglobin concentration between recovery interventions. Previously, CWI has been found to decrease blood myoglobin concentration compared to passive recovery (Bailey et al. 2007), TWI (Ascensão et al. 2011), and active recovery (Roberts et al. 2014). These results were found $30 \mathrm{~min}$ (Bailey et al. 2007), $60 \mathrm{~min}$ (Ascensão et al. 2011), and 2-6 h (Roberts et al. 2014) after exercise. However, after $24-48 \mathrm{~h}$, there were no differences between CWI and passive recovery (Bailey et al. 2007) or TWI (Ascensão et al. 2011).

The cytokine monocyte chemoattractant protein-1 (MCP1 ) is a sensitive marker of inflammation that increases immediately after muscle-damaging exercise (Peake et al. 2005). MCP-1 is necessary for the recruitment of monocytes for phagocytosis and facilitates repair of damaged myocytes (Chazaud et al. 2009; Lu et al. 2011). MCP-1 production is either constitutive or inducible by oxidative stress, cytokines or growth factors (Deshmane et al. 2009). To promote skeletal muscle repair, MCP-1 signalling up-regulates IGF-1 expression via intramuscular macrophages (Lu et al. 2011); indeed, MCP-1 deficient mice display impaired skeletal muscle regeneration (Shireman et al. 2007; Lu et al. 2011). Thus, accelerating return of MCP-1 concentrations to baseline after exercise may negatively impact training adaptation (Crystal et al. 2013). On the other hand, a reduction in MCP-1 release may be beneficial for short-term recovery, because it may inhibit the secondary damage arising as a result of inflammation following exercised-induced muscle damage (Crystal et al. 2013) and thus it could allow higher training frequency for possible better adaptation and performance during a tight competition season. However, in the present study MCP-1 concentration was unaffected by the recovery intervention. Only a few studies have investigated the effect of water immersion methods on MCP-1. In line with the present study, Crystal et al. (2013) did not find a difference in plasma MCP-1 concentration between CWI and passive recovery, but they observed a trend towards lower MCP-1 in the CWI group $6 \mathrm{~h}$ after exercise. Similarly, Peake et al. (2017) did not find a difference in MCP-1 expression between CWI and active recovery at 2, 24, and $48 \mathrm{~h}$ after exercise. These studies used different loading and recovery protocols compared to the present study. The present study and Peake et al. (2017) used $10{ }^{\circ} \mathrm{C}$ water temperature and 10 min immersion time in CWI. However, Crystal et al. (2013) used $5{ }^{\circ} \mathrm{C}$ water temperature and 20 min immersion time, resulting in higher cooling capacity. It has been suggested that CWI protocols of greater cooling capacity 
Table 1 Intraclass correlation coefficients for biomarkers measured at PRE and POST5 and the response magnitude from PRE to POST5 across all four trials. *, p < 0.05, **, p < $0.01, * * *, p<0.001$ with ICC $=0$ as reference

\begin{tabular}{llll}
\hline Variable & ICC pre & ICC post & ICC response magnitude \\
\hline MCP-1 & $0.718^{* * *}(0.440-0.914)$ & $0.659^{* * *}(0.359-0.892)$ & $0.139(-0.109$ to 0.576$)$ \\
CK & $0.555^{* * *}(0.220-0.851)$ & $0.338^{*}(0.029-0.732)$ & $0.065(-0.131$ to 0.481$)$ \\
hs-CRP & $0.353^{*}(0.043-0.878)$ & $0.388^{* *}(0.071-0.762)$ & $0.402^{* *}(0.084$ to 0.771$)$ \\
COR & $0.322^{* *}(0.045-0.710)$ & $0.556^{* * *}(0.232-0.848)$ & $0.609^{* * *}(0.298$ to 0.872$)$ \\
Myoglobin & $0.660^{* * *}(0.357-0.892)$ & $0.376^{* * *}(0.075-0.746)$ & $0.277^{* * *}(0.028$ to 0.664$)$ \\
\hline
\end{tabular}

Table 2 Intraclass correlation coefficients for white blood cells measured at PRE and POST5 and the response magnitude from PRE to POST5 across all four trials. *, p $<0.05, * *, p<0.01, * * *, p<0.001$ with ICC $=0$ as reference

\begin{tabular}{llll}
\hline Variable & ICC pre & ICC post & ICC response magnitude \\
\hline Leukocytes & $0.587^{* * *}(0.262-0.864)$ & $0.463^{* * *}(0.130-0.806)$ & $0.238(-0.036$ to 0.657$)$ \\
Lymphocytes & $0.463^{* *}(0.139-0.804)$ & $0.536^{* * *}(0.194-0.843)$ & $0.548^{* * *}(0.230-0.845)$ \\
Mixed cells & $0.215(-0.059$ to 0.643$)$ & $0.248(-0.046$ to 0.673$)$ & $-0.034(-0.234$ to 0.408$)$ \\
Neutrophils & $0.659^{* * *}(0.354-0.893)$ & $0.664 * * *(0.366-0.894)$ & $0.240^{*}(0.006-0.633)$ \\
\hline
\end{tabular}

(i.e., of longer duration or colder water temperature) may exacerbate the inflammatory response in the circulation (Lee et al. 2012; White et al. 2014), because cold water imposes a compound stress on the body (White et al. 2014). Therefore, it seems that CWI does not affect MCP-1 expression even if the cooling capacity varies. In the future, the effects of water immersion methods on MCP-1 expression could be studied using team sport and endurance exercise protocols, because CWI has been found to promote the recovery of performance and clearance of blood myoproteins after exercise with higher metabolic demands and longer durations compared to this study (Ihsan et al. 2016). However, from the results of the present study, it seems like CWI does not accelerate the return of MCP- 1 concentrations to baseline after muscle-damaging jumping and sprinting exercise.

In line with several previous studies (Pournot et al. 2011; Broatch et al. 2014; de Andrade Bezerra et al. 2014), we observed no differences in leukocytes and their subgroups (neutrophils, lymphocytes and mixed cells) between the four recovery methods. On the contrary, Stacey et al. (2010) found that after CWI, leukocyte and neutrophil counts were higher and lymphocyte counts were lower compared to both active and passive recovery. Stacey et al. (2010) used a similar recovery protocol in $\mathrm{CWI}\left(10^{\circ} \mathrm{C}, 10 \mathrm{~min}\right)$ to both previous studies and the present study $\left(10{ }^{\circ} \mathrm{C}, 10-15 \mathrm{~min}\right)$ (Pournot et al. 2011; Broatch et al. 2014; de Andrade Bezerra et al. 2014). However, in the study by Stacey et al. (2010) the loading protocol (high-intensity cycling) was metabolically more demanding, which might explain the observed different response. One plausible explanation for the fact that the present study did not observe significant differences between recovery methods could have been the loading protocol. It may be considered whether the loading protocol was sufficiently stressful to highlight the differences between the recovery methods. Cortisol levels, previously reported in Ahokas et al (2019), did not increase after the loading protocol, suggesting that as expected stress caused by exercise was low. It is possible that the exercise protocol may not be demanding enough to induce high levels of inflammation, where differences in recovery methods would be measured. Nevertheless, the recovery protocol was designed to match possible recovery routines used by athletes, which is where the present study differs from previous studies, and is beneficial for external validity. It should also be taken into account, that in the present study the participants were physically active men, but not athletes. It has been noted previously that physiological responses may differ between high level athletes and active men (Ahtiainen et al. 2003).

To avoid the repeated bout effect of the inflammatory response to exercise that has been previously illustrated following muscle-damaging eccentric exercise bouts (McHugh 2003), the participants took part into familiarisation session and a randomised design was used. Nevertheless, post-hoc reliability and repeatability analyses of biomarker concentrations at timepoints that were reproduced in all four trials suggested that the reliability of biomarker responses to exercise loading was low. Applied in the present context, the ICC essentially represents a ratio of within-subject variation to between-subject variation (Weir 2005). Typically, ICCs below 0.5 have been defined as poor reliability (Koo and $\mathrm{Li}$ 2016), and in the present study only lymphocytes displayed ICCs for the response magnitude to exercise loading above this threshold. Cortisol, previously reported in Ahokas et al (2019), also displayed a satisfactory ICC for the response to exercise, but it is, however, notable that cortisol was also the only biomarker that did not increase significantly in response to loading. Moreover, trial-order effects were evident for myoglobin and neutrophils, indicative of a blunting in the 
exercise response across the four trials, particularly from trial 1. This suggests that the single familiarisation trial was not sufficient to account for the 'repeated bout effect' for these markers. If we were to hypothesise that low reliability of biomarker responses extends to timepoints after the different interventions in each trial, we could suggest that subtle influences of recovery measures on the rate of resolution of inflammation may have been masked by high intra-individual variability. Although main effects of time during recovery were detected, with many biomarkers displaying a clear time-course response after loading and recovery, it is possible that due to variability in exercise-induced responses, a greater number of participants may have been required to detect interaction effects between recovery interventions over time. This critique could be extended to previous studies that have aimed to address similar research questions, which typically recruit similar numbers of participants to the present study (Stacey et al. 2010; Crystal et al. 2013; Broatch et al. 2014). High-quality studies are needed to perform meta-analytical analyses. Meta-analytical analyses of multiple studies performed under similar conditions may help to account for low statistical power in individual studies; indeed recent meta-analytical findings suggest that CWI may accelerate resolution of post-exercise inflammation despite individual studies often showing no effects (Higgins et al. 2017). A strength of this study was the use of a repeated-measures crossover approach, which helped to mitigate the limitation of a small sample size, though to a lesser extent for markers with low ICCs. Furthermore, the water immersion methods were not compared only to passive recovery, but the purpose was to investigate the added benefit of the water immersion methods compared to standard recovery routines frequently used by athletes.

In conclusion, the results of this study support the hypothesis that water immersion methods do not accelerate recovery from loading exercise via anti-inflammatory effects when combined with an active recovery protocol. It is important to acknowledge that the exploratory descriptive analysis of the reliability of the exercise-induced response to a standardised loading protocol in the present study showed that there was a low reliability of biomarker responses to a standardised loading exercise bout, even after an initial familiarisation trial. Thus, future studies with higher numbers of participants, improved familiarisation to loading exercise, or meta-analytic analyses are needed to clarify this issue. Future studies should take into account potential adaptation effects when performing repeated loading exercise and ensure sufficient time for wash-out.

Acknowledgements The present research was supported by Ecomarine Oy and Avantopool Oy. The authors would also like to thank the Emil Aaltonen foundation, and the Faculty of Sport and Health Sciences for financial contributions to this work. In addition, a heartfelt thank you is extended to the Faculty of Sport and Health Sciences' technical staff and students, as well as to the committed participants that participated in this study.

Author contributions EKA, HK, AAM, SW and JKI conceived and designed research. EKA and JKI conducted experiments. EKA, HGH, and JKI analysed data. All authors contributed to writing the manuscript. All authors have approved the final manuscript.

Funding Ecomarine Oy and Avantopool Oy, The Emil Aaltonen Foundation and the Faculty of Sport and Health Sciences made financial contributions to this work. Open access funding provided by University of Jyväskylä (JYU).

Availability of data and material Data is available on request.

\section{Compliance with ethical standards}

Conflict of interest None of the authors have a conflict of interest to declare.

Ethics approval The University of Jyväskylä Ethical Committee approved the study and included procedures/analyses.

Consent to participate Participants gave their written informed consent to take part in the present investigation.

Consent for publication The present authors all consent to the publication of this work.

Open Access This article is licensed under a Creative Commons Attribution 4.0 International License, which permits use, sharing, adaptation, distribution and reproduction in any medium or format, as long as you give appropriate credit to the original author(s) and the source, provide a link to the Creative Commons licence, and indicate if changes were made. The images or other third party material in this article are included in the article's Creative Commons licence, unless indicated otherwise in a credit line to the material. If material is not included in the article's Creative Commons licence and your intended use is not permitted by statutory regulation or exceeds the permitted use, you will need to obtain permission directly from the copyright holder. To view a copy of this licence, visit http://creativecommons.org/licenses/by/4.0/.

\section{References}

Ahokas EK, Ihalainen JK, Kyröläinen H, Mero AA (2019) Effects of water immersion methods on postexercise recovery of physical and mental performance. J Strength Cond Res 33:1488-1495. https://doi.org/10.1519/JSC.0000000000003134

Ahtiainen JP, Pakarinen A, Alen M et al (2003) Muscle hypertrophy, hormonal adaptations and strength development during strength training in strength-trained and untrained men. Eur J Appl Physiol 89:555-563. https://doi.org/10.1007/s00421-003-0833-3

Ascensão A, Leite M, Rebelo AN et al (2011) Effects of cold water immersion on the recovery of physical performance and muscle damage following a one-off soccer match. J Sports Sci 29:217225. https://doi.org/10.1080/02640414.2010.526132

Bailey DM, Erith SJ, Griffin PJ et al (2007) Influence of cold-water immersion on indices of muscle damage following prolonged intermittent shuttle running. J Sports Sci 25:1163-1170. https:// doi.org/10.1080/02640410600982659 
Bleakley C, McDonough S, Gardner E et al (2012) Cold-water immersion (cryotherapy) for preventing and treating muscle soreness after exercise. Cochrane Database Syst Rev. https://doi. org/10.1002/14651858.cd008262.pub2

Broatch JR, Petersen A, Bishop DJ (2014) Postexercise cold water immersion benefits are not greater than the placebo effect. Med Sci Sports Exerc 46:2139-2147. https://doi.org/10.1249/ MSS.0000000000000348

Brophy-Williams N, Landers G, Wallman K (2011) Effect of immediate and delayed cold water immersion after a high intensity exercise session on subsequent run performance. J Sport Sci Med 10:665670. https://doi.org/10.1016/j.jsams.2011.11.238

Brouwer N, van Pelt J (2015) Validation and evaluation of eight commercially available point of care CRP methods. Clin Chim Acta 439:195-201. https://doi.org/10.1016/j.cca.2014.10.028

Brunelli S, Rovere-Querini P (2008) The immune system and the repair of skeletal muscle. Pharmacol Res 58:117-121. https:// doi.org/10.1016/j.phrs.2008.06.008

Camargo MZ, Siqueira CPCM, Preti MCP et al (2012) Effects of light emitting diode (LED) therapy and cold water immersion therapy on exercise-induced muscle damage in rats. Lasers Med Sci 27:1051-1058. https://doi.org/10.1007/s10103-011-1039-2

Chazaud B, Brigitte M, Yacoub-Youssef H et al (2009) Dual and beneficial roles of macrophages during skeletal muscle regeneration. Exerc Sport Sci Rev 37:18-22

Cote DJ, Prentice WE, Hooker DN, Shields EW (1988) Comparison of three treatment procedures for minimizing ankle sprain swelling. Phys Ther 68:1072-1076. https://doi.org/10.1093/ $\mathrm{ptj} / 68.7 .1072$

Crystal NJ, Townson DH, Cook SB, Laroche DP (2013) Effect of cryotherapy on muscle recovery and inflammation following a bout of damaging exercise. Eur J Appl Physiol 113:2577-2586. https://doi.org/10.1007/s00421-013-2693-9

de Andrade Bezerra J, de Castro ACM, Melo SVA et al (2014) Passive, active, and cryotherapy post-match recovery strategies induce similar immunological response in soccer players. Int J Sports Sci 4(6A):12-18. https://doi.org/10.5923/s.sports.20140 1.02

Deshmane SL, Kremlev S, Amini S, Sawaya BE (2009) Monocyte chemoattractant protein-1 (MCP-1): an overview. J. Interf. Cytokine Res. 29:313-325

Dupuy O, Douzi W, Theurot D et al (2018) An evidence-based approach for choosing post-exercise recovery techniques to reduce markers of muscle damage, Soreness, fatigue, and inflammation: a systematic review with meta-analysis. Front Physiol 9:403. https://doi.org/10.3389/fphys.2018.00403

Eston R, Peters D (1999) Effects of cold water immersion on the symptoms of exercise-induced muscle damage. J Sports Sci 17:231-238. https://doi.org/10.1080/026404199366136

Freidenreich DJ, Volek JS (2012) Immune responses to resistance exercise. Exerc Immunol Rev 18:8-41

Higgins TR, Greene DA, Baker MK (2017) Effects of cold water immersion and contrast water therapy for recovery from team sport: a systematic review and meta-analysis. J Strength Cond Res 31:1443-1460

Ihsan M, Watson G, Abbiss CR (2016) What are the Physiological Mechanisms for Post-Exercise Cold Water Immersion in the Recovery from Prolonged Endurance and Intermittent Exercise? Sport Med 46:1095-1109

Ingram J, Dawson B, Goodman C et al (2009) Effect of water immersion methods on post-exercise recovery from simulated team sport exercise. J Sci Med Sport 12:417-421. https://doi. org/10.1016/j.jsams.2007.12.011

Koo TK, Li MY (2016) A Guideline of Selecting and Reporting Intraclass Correlation Coefficients for Reliability Research.
J Chiropr Med 15:155-163. https://doi.org/10.1016/j. jcm.2016.02.012

Lee EC, Watson G, Casa D et al (2012) Interleukin-6 responses to water immersion therapy after acute exercise heat stress: a pilot investigation. J Athl Train 47:655-663. https://doi. org/10.4085/1062-6050-47.5.09

Lu H, Huang D, Ransohoff RM, Zhou L (2011) Acute skeletal muscle injury: cCL2 expression by both monocytes and injured muscle is required for repair. FASEB J 25:3344-3355. https:// doi.org/10.1096/fj.10-178939

Mawhinney C, Jones H, Low DA et al (2017) Influence of coldwater immersion on limb blood flow after resistance exercise*. Eur J Sport Sci 17:519-529. https://doi.org/10.1080/17461 391.2017.1279222

McHugh MP (2003) Recent advances in the understanding of the repeated bout effect: the protective effect against muscle damage from a single bout of eccentric exercise. Scand J Med Sci Sport 13:88-97

Murray A, Cardinale M (2015) Cold applications for recovery in adolescent athletes: a systematic review and meta analysis. Extrem Physiol Med 4:1-15

Peake JM, Suzuki K, Hordern M et al (2005) Plasma cytokine changes in relation to exercise intensity and muscle damage. Eur J Appl Physiol 95:514-521. https://doi.org/10.1007/s0042 1-005-0035-2

Peake J, Della Gatta P, Cameron-Smith D (2010) Aging and its effects on inflammation in skeletal muscle at rest and following exercise-induced muscle injury. Am J Physiol Regul Integr Comp, Physiol, p 298

Peake JM, Roberts LA, Figueiredo VC et al (2017) The effects of cold water immersion and active recovery on inflammation and cell stress responses in human skeletal muscle after resistance exercise. J Physiol 595:695-711. https://doi.org/10.1113/JP272 881

Pointon M, Duffield R, Cannon J, Marino FE (2012) Cold water immersion recovery following intermittent-sprint exercise in the heat. Eur J Appl Physiol 112:2483-2494. https://doi. org/10.1007/s00421-011-2218-3

Pournot H, Bieuzen F, Duffield R et al (2011) Short term eVects of various water immersions on recovery from exhaustive intermittent exercise. Eur J Appl Physiol 111:1287-1295. https://doi. org/10.1007/s00421-010-1754-6

Roberts LA, Nosaka K, Coombes JS, Peake JM (2014) Cold water immersion enhances recovery of submaximal muscle function after resistance exercise. Am J Physiol Regul Integr Comp Physiol 307:R998-R1008. https://doi.org/10.1152/ajpregu.00180 .2014

Shireman PK, Contreras-Shannon V, Ochoa O et al (2007) MCP-1 deficiency causes altered inflammation with impaired skeletal muscle regeneration. J Leukoc Biol 81:775-785. https://doi. org/10.1189/jlb.0506356

Stacey DL, Gibala MJ, Martin Ginis KA, Timmons BW (2010) Effects of recovery method after exercise on performance, immune changes, and psychological outcomes. J Orthop Sports Phys Ther 40:656-665. https://doi.org/10.2519/jospt.2010.3224

Vaile JM, Gill ND, Blazevich AJ (2007) The effect of contrast water therapy on symptoms of delayed onset muscle soreness. J Strength Cond Res 21:697-702. https://doi. org/10.1519/R-19355.1

Versey NG, Halson SL, Dawson BT (2013) Water immersion recovery for athletes: effect on exercise performance and practical recommendations. Sport Med 43:1101-1130

Wackerhage H, Schoenfeld BJ, Hamilton DL et al (2019) Stimuli and sensors that initiate skeletal muscle hypertrophy following resistance exercise. J Appl Physiol 126:30-43. https://doi. org/10.1152/japplphysiol.00685.2018 
Warren GL, Lowe DA, Armstrong RB (1999) Measurement tools used in the study of eccentric contraction-induced injury. Sport Med 27:43-59. https://doi.org/10.2165/00007256-19992 7010-00004

Weir JP (2005) Quantifying test-retest reliability using the intraclass correlation coefficient and the SEM. J Strength Cond Res 19:231-240. https://doi.org/10.1519/15184.1

White GE, Rhind SG, Wells GD (2014) The effect of various coldwater immersion protocols on exercise-induced inflammatory response and functional recovery from high-intensity sprint exercise. Eur J Appl Physiol 114:2353-2367. https://doi. org/10.1007/s00421-014-2954-2

Wilcock IM, Cronin JB, Hing WA (2006) Physiological response to water immersion: a method for sport recovery? Sport Med $36: 747-765$

Yamane M, Teruya H, Nakano M et al (2006) Post-exercise leg and forearm flexor muscle cooling in humans attenuates endurance and resistance training effects on muscle performance and on circulatory adaptation. Eur J Appl Physiol 96:572-580. https:// doi.org/10.1007/s00421-005-0095-3

Yamane M, Ohnishi N, Matsumoto T (2015) Does regular post-exercise cold application attenuate trained muscle adaptation? Int J Sports Med 36:647-653. https://doi.org/10.1055/s-0034-13986 52

Yanagisawa O, Niitsu M, Takahashi H et al (2003) Evaluations of cooling exercised muscle with MR imaging and 31P MR spectroscopy. Med Sci Sports Exerc 35:1517-1523. https://doi.org/10.1249/01. MSS.0000084418.96898.2E

Publisher's Note Springer Nature remains neutral with regard to jurisdictional claims in published maps and institutional affiliations. 